\title{
Regional lung function and heterogeneity of specific gas volume in healthy and emphysematous subjects
}

\author{
Andrea Aliverti*, Francesca Pennati*, Caterina Salito* and Jason C. Woods, ${ }^{\#, ~}$
}

ABSTRACT: The aim of our study was to study regional lung function by standard computed tomography (CT) and characterise regional variations of density and specific gas volume (SVg) between different lung volumes.

We studied 10 healthy and 10 severely emphysematous subjects. Corresponding CT images taken at high and low lung volumes were registered by optical flow to obtain two-dimensional maps of pixel-by-pixel differences of density $(\Delta \mathrm{HU})$ and $\mathrm{SVg}(\Delta \mathrm{SVg})$ at slice levels near the aortic arch, carina and top diaphragm.

In healthy subjects, $\Delta \mathrm{HU}$ was higher at all levels $(\mathrm{p}<0.001)$ with higher variability expressed as interquartile range $(p<0.001)$, largely due to its differences between dorsal and ventral regions. In patients, median $\Delta \mathrm{SVg}$ values were 3.2 times lower than healthy volunteers $(p<0.001)$, while heterogeneity of $\Delta \mathbf{S V g}$ maps, expressed as quartile coefficient of variation, was 5.4 times higher $(p<0.001)$. In all patients, there were areas with negative values of $\Delta \mathbf{S V g}$.

In conclusion, $\Delta \mathbf{S V g}$ is uniform in healthy lungs and minimally influenced by gravity. The significant $\Delta \mathbf{S V g}$ heterogeneity observed in emphysema allows identification of areas of alveolar destruction and gas trapping and suggests that $\Delta \mathrm{SVg}$ maps provide useful information for evaluation and planning of emerging treatments that target trapped gas for removal.

\section{KEYWORDS: Emphysema, high-resolution computed tomography, image analysis}

$\mathrm{n}$ recent years, a number of different bronchoscopic techniques have been introduced for the treatment of severe emphysema, such as airway bypass [1, 2], endobronchial one-way exit valves [3], thermal vapour ablation [4], biological sealants [5, 6] and airway implants [7]. Functional regional analysis of the lung is required as a tool for planning and guiding these treatments. Nuclear imaging, such as single photon emission computed tomography (CT) and positron emission tomography, provides direct regional ventilation imaging [8-10], but suffers from low spatial resolution and low signal-to-noise ratio. Hyperpolarised noble gas magnetic resonance imaging is a safe technique that provides regional ventilation imaging with no repeated exposure to ionising radiation and with good temporal resolution [11-13]. Conversely, it suffers from being partially quantitative and from requiring special equipment to hyperpolarise the gas. Xenonenhanced CT provides a regional measure of ventilation with high spatial resolution. Xenon gas, however, is expensive, requires special equipment and must be carefully monitored because of its sedative and anaesthetic properties [14, 15].
Recently, standard CT has been increasingly considered not only to study parenchymal and airway wall anatomical alterations in emphysema, but also to provide data on regional lung function by using images acquired at different lung volumes. SIMON [16] introduced the concept of specific volume change between corresponding regions at inspired and expired breath-hold CT images. DOUGHERTY and co-workers [17, 18] proposed an innovative method to visualise gas trapping in emphysema based on the density changes occurring between registered lung volumes.

CoxsON et al. [19] introduced the concept of specific gas volume (SVg), i.e. the volume of gas per gramme of lung tissue. More recently, SALITO et al. [20] demonstrated how the analysis of the variations of $\mathrm{SVg}$ can provide a valuable tool for clearly identifying and quantifying the extent and severity of trapped gas. The same authors have also shown that the use of thick slices and a smooth filter for image reconstruction reduce the effect of low-attenuation pixels on SVg that leads to overestimation of the severity of emphysema and trapped gas [21].

\section{AFFILIATIONS}

*TBMLab Dipartimento di Elettronica, Informazione e Bioingegneria, Politecnico di Milano, Milan, Italy, \#Dept of Radiology, Washington University, St. Louis, and "Dept of Physics, Washington University, St. Louis, MO, USA.

CORRESPONDENCE

A. Aliverti

TBMLab

Dipartimento di Elettronica Informazione e Bioingegneria Politecnico di Milano

Via G. Colombo 40 20133 Milano Italy

E-mail: andrea.aliverti@polimi.it

Received:

March 232012

Accepted after revision: July 242012 First published online: Aug 092012 
We hypothesised that differences in SVg obtained from registered CT images acquired at two different lung volumes, visualised as coloured maps and quantified as frequency distributions, are able to provide regional functional evaluation in severe emphysema. More specifically, the aims of the present work were to: 1) introduce a new method of registration and analysis able to regionally investigate lung function in terms of SVg variations, based on standard CT images acquired at two different lung volumes; 2 ) evaluate the proposed method in both healthy volunteers and subjects with severe emphysema; and 3) explore possible mechanisms contributing to different patterns of SVg heterogeneity, such as gravity and collateral ventilation.

\section{MATERIALS AND METHODS Study subjects}

CT imaging on 10 healthy volunteers with no history of smoking or lung disease and on 10 patients with severe emphysema (forced expiratory volume in $1 \mathrm{~s}<50 \%$ predicted, residual volume $(\mathrm{RV}) /$ total lung capacity $(\mathrm{TLC}) \geqslant 0.65)$ were performed for this study. The Institutional Review Board of the Washington University in St. Louis, MO, USA, approved the protocol for healthy subjects and informed written consent was obtained from each one. The data collected on emphysematous patients originated from different locations and are part of the pre-treatment assessment database of a clinical trial evaluating the safety and effectiveness of a new procedure called airway bypass (registered at www.clinicaltrials.gov with identifier number NCT00391612). Local ethical committee review and approval were obtained, and written informed consent was obtained from all the patients.

Healthy volunteers and patients were scanned while supine during suspended end-inspiration at TLC and during suspended end-expiration at RV.

CT scans of all the healthy subjects were performed using a SOMATOM Definition Dual Source CT (Siemens, Forchheim, Germany). Scanner settings were as follows: tube voltage, $120 \mathrm{kVp}$; tube current, $110 \mathrm{~mA}$; rotation time, $500 \mathrm{~ms}$; matrix, $512 \times 512$; slice reconstruction thickness, $5 \mathrm{~mm}$. In patients with emphysema, CT imaging (rotation time, $500 \mathrm{~ms}$; matrix, $512 \times 512$; slice reconstruction thickness, $10 \mathrm{~mm}$ ) was performed using the same type of Siemens scanner in nine subjects and using a GE scanner (Light-Speed VCT; GE Healthcare, Milwaukee, WI, USA) in one patient (rotation time $741 \mathrm{~ms}$ ). CT images were reconstructed with B30f and "standard" reconstruction filters, respectively, for Siemens and GE scanners. The resulting radiation dose was in the order of 2.4 and $2.5-2.7 \mathrm{mSv}$ per scan, respectively, in both healthy controls and patients.

\section{Image analysis}

The method for image segmentation, registration and warping is summarised in fig. 1. From the scans acquired at TLC and $\mathrm{RV}$, the correspondent images at the same apical-basal level of the lung were selected. As suggested by Mishima et al. [22], three slice-levels were considered: aortic arch (AA), carina (C) and top diaphragm (TD). At each of these levels, images taken at RV were deformed onto the TLC images by an automatic registration algorithm based on the optical flow method (OFM) proposed by LUCAS and KANADE [23].
Successively, the inspiratory and deformed expiratory CT images were converted into both Hounsfield Units (HU) and $\mathrm{SVg}$ [20] and then subtracted on a pixel-by-pixel basis to evaluate the HU and $\mathrm{SVg}$ difference maps ( $\Delta \mathrm{HU}$ and $\Delta \mathrm{SVg}$, respectively) between the two lung volumes. SVg was calculated pixel-by-pixel as:

$$
\mathrm{SVg}=\text { specific volume }_{(\text {tissue }} \text { and gas) } \text {-specific volume } \text { (tissue) }
$$

where specific volume (expressed in $\mathrm{mL} \cdot \mathrm{g}^{-1}$ ) is the inverse of density $\left(\mathrm{g} \cdot \mathrm{mL}^{-1}\right)$.

The specific volume of the lung (tissue and gas) was measured from the CT as:

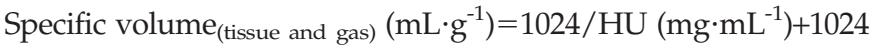

On the basis of existing literature [24], the specific volume of tissue was assumed to be equal to $1 / 1.065=0.939 \mathrm{~mL} \cdot \mathrm{g}^{-1}$.

Once the maps of $\Delta \mathrm{HU}$ and $\Delta \mathrm{SVg}$ were obtained at the AA, C and TD levels, descriptive statics were calculated.

\section{Image registration}

Image registration was performed by applying a procedure based on the following four steps. 1) Segmentation of the lung, based on the method proposed by $\mathrm{Hu}$ et al. [25]. 2) Preprocessing the input images to reduce any intensity bias by extraction of the following features from the original images: Laplacian [18], external border of the lung, vessels and fissures. Laplacian was obtained by a five-by-five image filter approximating the shape of the two-dimensional Laplacian operator. Vessels were extracted by thresholding the images at $-400 \mathrm{HU}$. Fissures were extracted semi-automatically on the basis of horizontal and vertical gradients of the image. The extracted features were combined into a single pre-processed image satisfying the requirements of standard OFM, i.e. conservation of grey intensity of the moving structures. 3) Estimation of the global transformation describing the global motion of the lung, including translation, rotation and dilation of the entire image. 4) Application of OFM to the pre-processed images to estimate the local deformation of the lung on a pixel-by-pixel basis. The OFM relies on the hypothesis of pixel grey intensity conservation under motion [26] and displacement vector similarity within a small neighbourhood surrounding the pixel [23]. To overcome the large displacement occurring between inspiratory and expiratory lung images, a pyramidal approach [27] organised in four levels was applied. From the coarsest to the finest level of the pyramid, each stage the motion was iteratively estimated to maximise the cross-correlation with the reference image. The resulting vector field, expressing the pixel-by-pixel displacement between the two images, was used to deform the expiratory lung image onto the inspiratory one. A linear interpolation was applied to maintain the grey level of the parenchyma.

\section{Quantitative analysis}

Once the registration process was completed, difference maps were obtained by subtracting Hounsfield units and SVg values pixel-by-pixel on the registered images.

$\Delta \mathrm{HU}$ was defined as:

$$
\Delta \mathrm{HU}=\mathrm{HU}_{\mathrm{RV}}-\mathrm{HU}_{\mathrm{TLC}}
$$




\section{Slice selection} RV

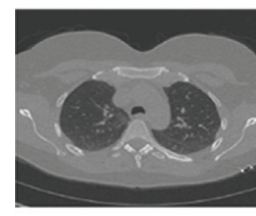

Segmentation

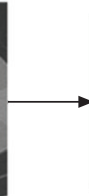

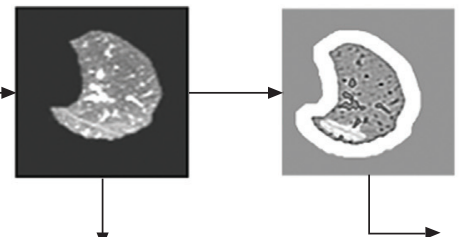

Warping
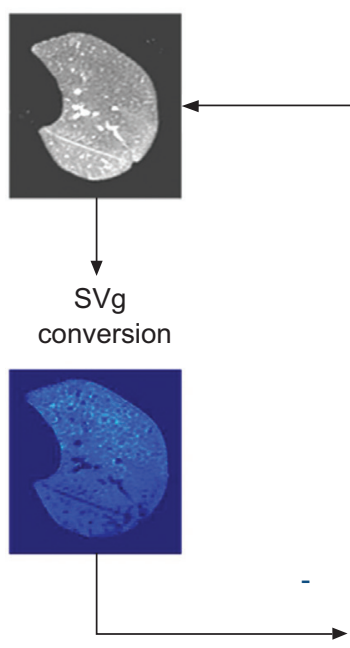

Pre-processing
Pre-processing

Segmentation

Slice selection TLC 


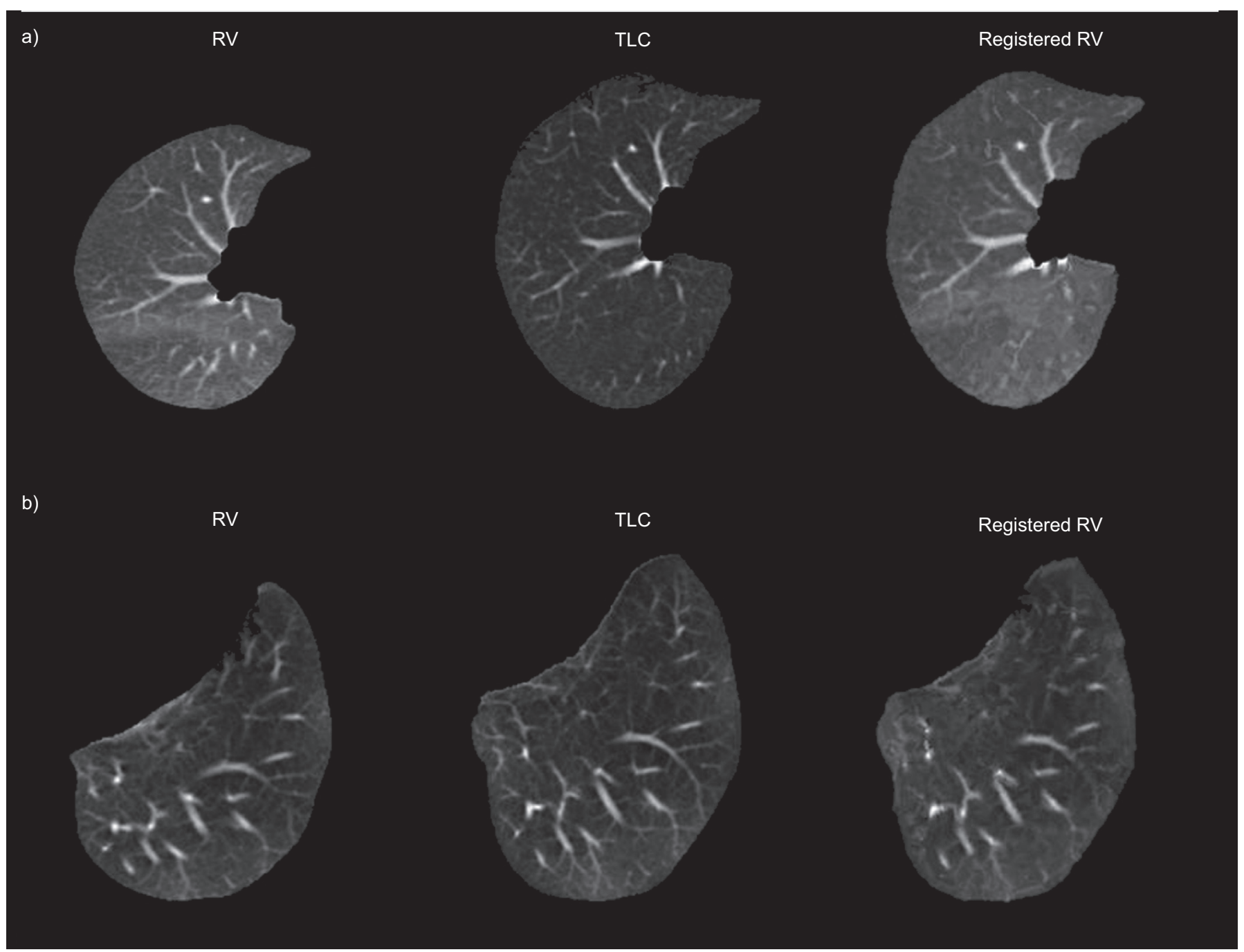

FIGURE 2. Representative results of image registration process on a) healthy and b) emphysematous lungs. In each panel, the segmented lung at residual volume (RV), total lung capacity (TLC) and the deformed RV image (registered RV) are shown.

Representative $\Delta \mathrm{HU}$ maps are shown in figure 3 from one healthy (fig. 3a) and one emphysematous (fig. 3b) subject. Maps at the three different considered lung levels are illustrated. In the healthy subject, at each level, $\Delta \mathrm{HU}$ is nonuniformly distributed within the lung, with increasing values from ventral to dorsal areas. In the patient with emphysema, the range of $\Delta H U$ variations is markedly reduced and no gradient is present in any direction at all lung levels.

The $\Delta S V g$ maps obtained from the same subjects shown in figure 3 are illustrated in figure 4 . While the healthy subject is characterised at every level by a homogeneous distribution of $\Delta S V g$ within the lung with $\Delta \mathrm{SVg}$ values averaging about $5 \mathrm{~mL} \cdot \mathrm{g}^{-1}$, the patient with emphysema shows a much higher degree of $\Delta \mathrm{SVg}$ heterogeneity and lower values averaging about $2 \mathrm{~mL} \cdot \mathrm{g}^{-1}$.

The individual histograms of $\Delta \mathrm{HU}$ and $\Delta \mathrm{SVg}$ of all healthy and emphysematous subjects for each lung level are shown in figure 5 and the results of descriptive statistics are shown in table 1 , and suggest interesting observations. Clearly, the $\Delta \mathrm{HU}$ and $\Delta S V g$ heterogeneity and average values described above and shown in figures 3 and 4 in two representative subjects were found to be common to the entire population of either healthy or emphysematous subjects. Emphysema was characterised by significantly lower median $\Delta \mathrm{HU}$ values that were distributed over a narrower range of values (lower IQR) compared with healthy subjects $(\mathrm{p}<0.001)$. Conversely, $\Delta \mathrm{SVg}$ was significantly lower in emphysematous than in healthy subjects $(p<0.001)$, and IQR was higher in emphysematous than in healthy subjects $(\mathrm{p}<0.001)$. Quartile variation coefficients $(\mathrm{QCV})$ of both $\Delta \mathrm{HU}$ and $\Delta \mathrm{SVg}$ were significantly higher $(\mathrm{p}<0.001)$ in emphysematous than in healthy subjects, suggesting a higher degree of heterogeneity. Healthy subjects are also characterised by left-skewed $\Delta \mathrm{SVg}$ histograms (i.e. a longer tail in the range of lower $\Delta S \mathrm{Vg}$ values and distribution mass concentrated on the range of higher $\Delta S V g$ values) associated with negative values of skewness, particularly at the AA and C levels. This means that in the healthy lung, the occurrence of high $\Delta \mathrm{SVg}$ values is more frequent than low $\Delta \mathrm{SVg}$ values. Conversely, severely emphysematous patients are characterised by right-skewed $\Delta \mathrm{SVg}$ distributions and positive skewness, suggesting more frequent low $\Delta \mathrm{SVg}$ values, independent of lung level. 
a) Healthy $\Delta \mathrm{HU}$

AA

C TD

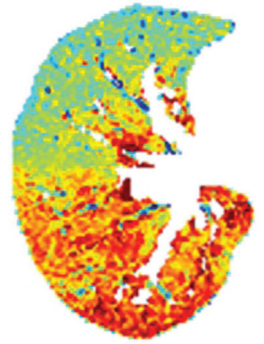

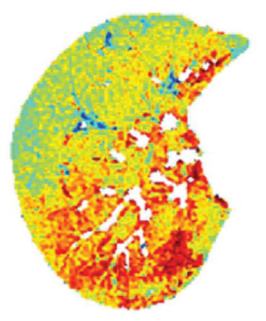
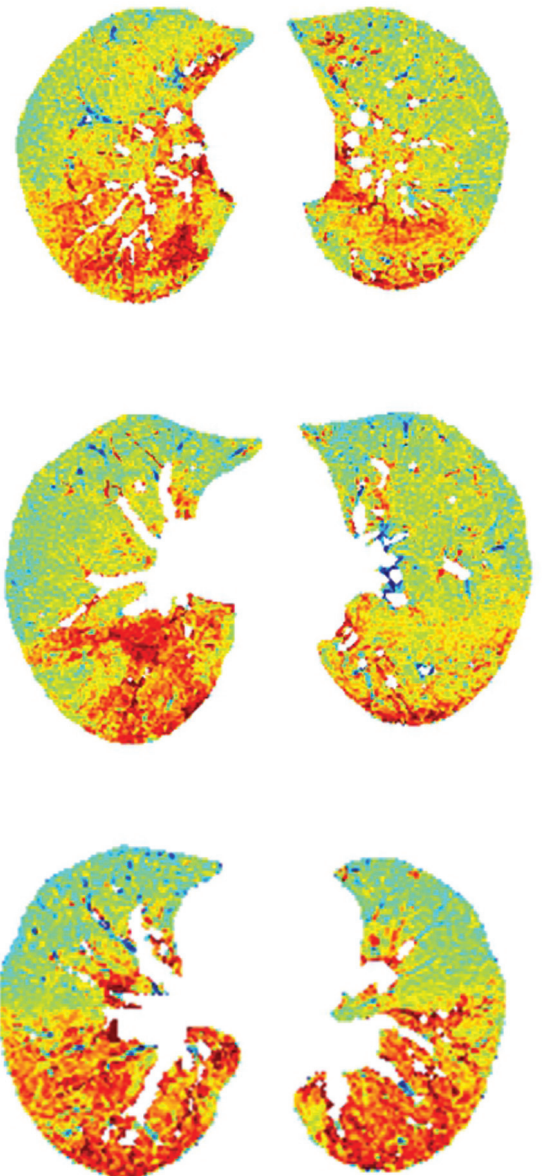

b)

Emphysema $\Delta \mathrm{HU}$

HU

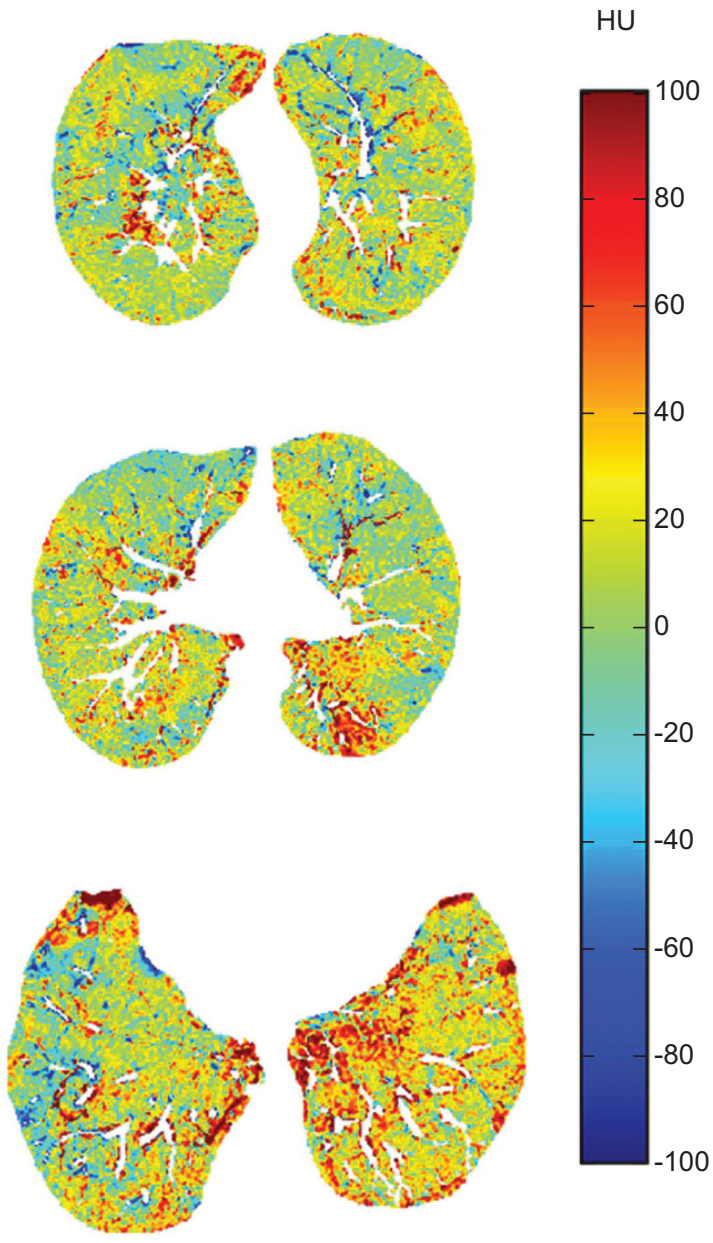

FIGURE 3. Hounsfield unit variations, calculated as $\Delta H U=H U_{R V}-H U_{T L C}$, where $\Delta H U$ is the difference in pixel density, $H U_{R V}$ is the pixel density at warped residual volume and $\mathrm{HU}_{\mathrm{TLC}}$ is the pixel density at total lung capacity of a representative a) healthy and b) emphysematous subject, calculated at aortic arch (AA), carina (C) and top of diaphragm (TD) levels. The ranges of display are chosen differently for the two groups (-100-300 HU for the healthy and -100-100 HU for the emphysematous lung) in order to highlight the changes occurring between the two lung volumes in emphysema, which is characterised by a lower range of variation.

The vertical (ventral to dorsal) variations of $\Delta \mathrm{HU}$ and $\Delta \mathrm{SVg}$, clearly visible in the representative subjects shown in figures 3 and 4 , were quantitatively analysed and the results are shown in figure 6. In healthy controls, $\Delta \mathrm{HU}$ was dependent on gravity, i.e. larger in dorsal than ventral regions, at all lung levels $(\mathrm{p}<0.01$ at AA level and $p<0.001$ at $C$ and TD levels). This effect was more pronounced at the TD level, where the central region was more significantly different than ventral $(\mathrm{p}<0.01)$ and dorsal $(\mathrm{p}<0.001)$ compared with the AA and C levels. Conversely, in emphysematous subjects, $\Delta \mathrm{HU}$, although different between levels, was not gravity dependent. No gravitational gradients of $\Delta \mathrm{SVg}$ were present both in healthy and emphysematous subjects.

As shown by the individual $\Delta \mathrm{SVg}$ histograms (fig. 5), in all patients with emphysema a number of negative $\Delta S V g$ values were present. In figure 7, a representative example of decreasing density from TLC to RV resulting in negative $\Delta S V g$ values is shown.

\section{DISCUSSION}

In this article, we propose a new method for the analysis of the regional lung function in terms of density and $\Delta \mathrm{SVg}$ between different lung volumes and we introduce innovative methods for registration of lung CT images taken at high and low lung volume. Our results demonstrate that the proposed methods can be successfully applied in both healthy subjects and patients with severe emphysema, for a qualitative and quantitative evaluation of tissue and gas changes with volume, which relate directly to lung function.

In healthy subjects, the higher within-subject variability of $\Delta \mathrm{HU}$ values, indicated by the higher IQR, is associated with the gravitational dependence of density within the lung, with the dorsal regions showing values of $\Delta \mathrm{HU}$ higher than the ventral ones (fig. 6). Conversely, patients with severe emphysema are characterised by $\Delta \mathrm{HU}$ values much lower than healthy controls distributed over a narrower range of values and associated with no gravity dependence (fig. 6). When maps of $\Delta \mathrm{SVg}$ are considered, no gravitational gradient is present in either healthy subjects or in subjects with emphysema (fig. 6). Healthy subjects are characterised by a homogeneous distribution of $\Delta \mathrm{SVg}$ at all the three slice levels (fig. 4a, c and e), as indicated by the lower value of $\mathrm{QVC}$. Conversely, $\mathrm{SVg}$ variations are markedly lower in 
a)

AA

C

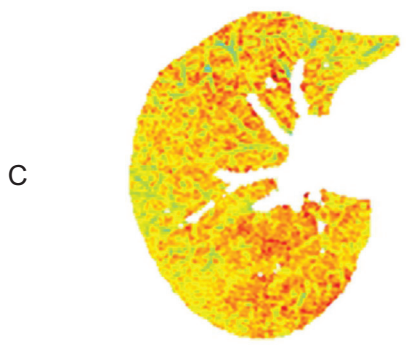

TD

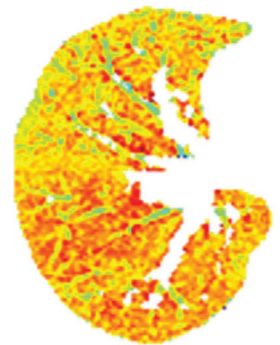

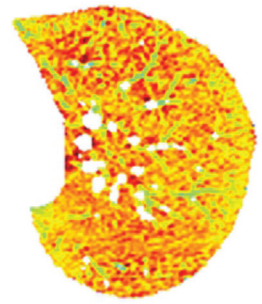
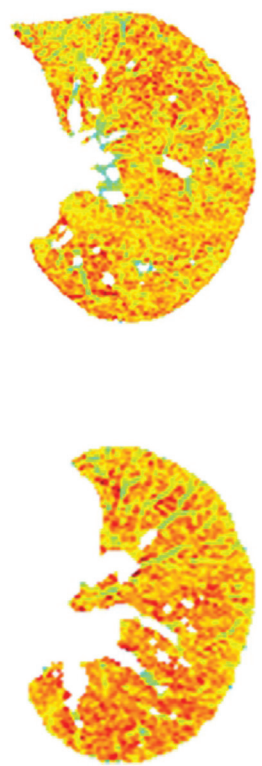

b)

Emphysema $\Delta S V g$

$\mathrm{mL} \cdot \mathrm{g}^{-1}$
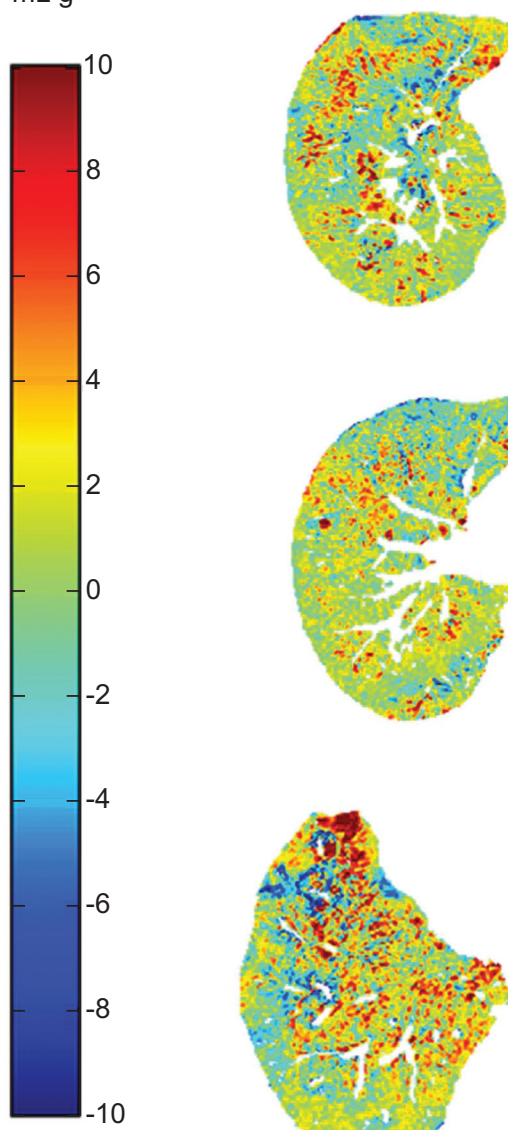

$\mathrm{mL} \cdot \mathrm{g}^{-1}$
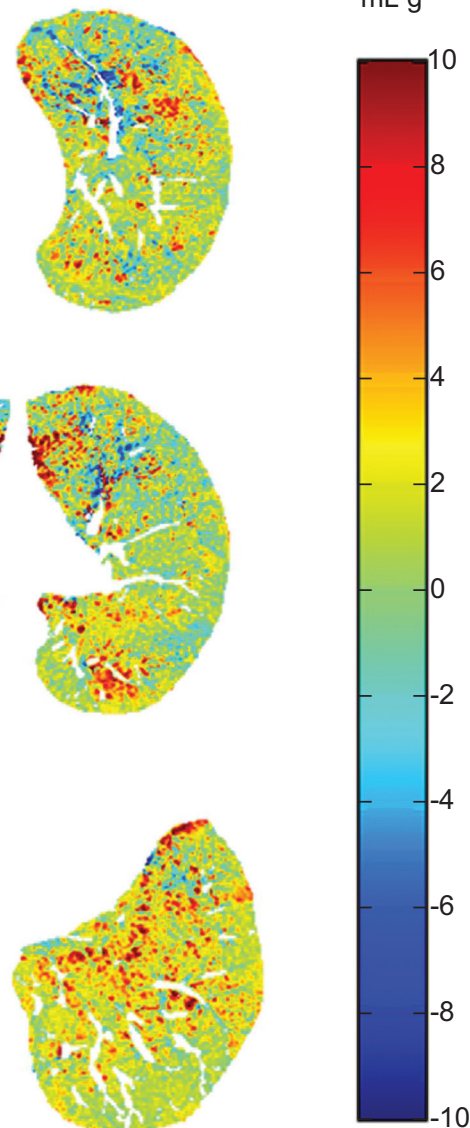

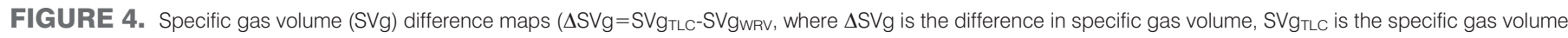

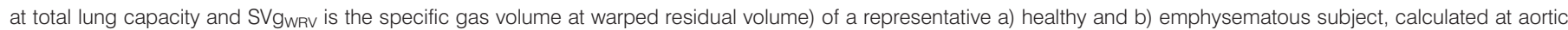
$\operatorname{arch}(A A)$, carina $(C)$ and top diaphragm (TD) levels. The chosen range of display is from -10 to $10 \mathrm{~mL} \cdot \mathrm{g}^{-1}$ in both the cases.

patients with severe emphysema and more heterogeneously distributed within the lung (fig. 4, right). Our findings suggest that alveolar destruction and gas trapping occur at all the considered levels in the emphysematous lung with, however, a high degree of local heterogeneity, and that QVC might provide an index to enable us to detect early signs of emphysema.

Our findings are in accordance with the classical studies of Milic-EMILI et al. [8] and BRYAN et al. [28] on regional ventilation. These authors showed that in the range of lung volumes between 20 and $100 \%$ of vital capacity, the proportion of inspired gas delivered to any lung region is constant, but gravity-dependent lung zones receive relatively more of the inspired volume than the nongravity-dependent zones.

In the current work, the $\Delta H U$ maps obtained in healthy subjects show how in the dependent (dorsal) regions gas volume changes are higher than in the nondependent (ventral) areas. In the dependent regions at RV, density is higher as alveoli are compressed, while at TLC density is reduced by alveolar opening. Conversely, the corresponding maps of $\Delta \mathrm{SVg}$ show that in the healthy lung, $\Delta \mathrm{SVg}$ is highly homogeneous and, therefore, the changes of the amount of gas relative to tissue mass are the same throughout the lung. The implication of these findings is that considering $\Delta \mathrm{SVg}$ maps rather than those reported by DOUGHERTY and coworkers [17, 18] or the ventilation maps proposed by GUERRERO and co-workers [29, 30], has the great advantage that the dependence of ventilation distribution on gravity is minimised. In these maps, therefore, any heterogeneity is the result of phenomena other than gravity. An interesting example is shown in figure 4 . The $\Delta S V g$ map at the TD level in the emphysematous patient shows a high local heterogeneity in the ventral part of the right lung. Positive (red) and negative (blue) $\Delta \mathrm{SVg}$ values alternate, suggesting that in this area, there are small regions where $\mathrm{SVg}$ increases, as expected, going from RV to TLC adjacent to small regions where $\mathrm{SVg}$ decreases. One possible explanation of this finding is the local heterogeneity in tissue compliance. Another possible mechanism is the presence of collateral channels that allow collateral flow in this area. Conversely, in the healthy lung, collateral ventilation has a negligible importance in the distribution of ventilation as the collateral channels have about 50 times higher resistance than the normal airways [31]. HoGG et al. [31] revealed a decreased resistance to collateral airflow in the post 

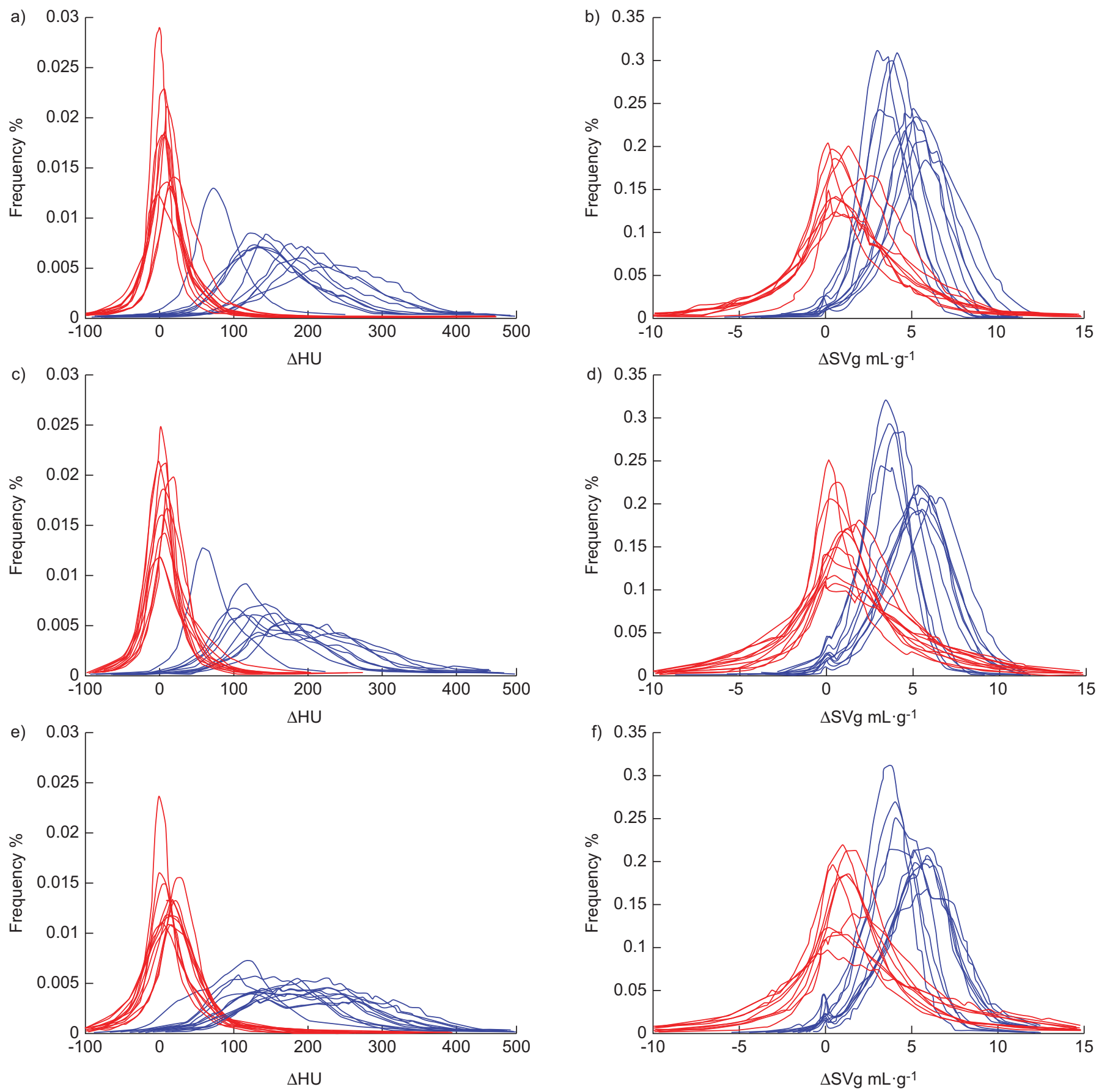

FIGURE 5. a, c, e) The probability distribution of the change in Hounsfield units $(\Delta H U)$ and b, d, f) change in specific gas volume ( $\Delta S V g)$ values of the three levels of the lung: $\mathrm{a}, \mathrm{b}$ ) aortic arch; c, d) carina; e, f) top of diaphragm. In each graph, the healthy subjects (blue) are characterised by higher $\Delta \mathrm{HU}$ and $\Delta \mathrm{SV} g$ values, as the histograms are shifted towards higher values. The interquartile range of the healthy results higher in $\Delta \mathrm{HU}$ but lower in $\Delta \mathrm{SVg}$ with respect to emphysema, and this behaviour could be explained by the gravity effect to which the healthy maps are subjected. Moreover, in emphysema, these histograms show a variable percentage of negative values that could be explained as a different percentage of collateral ventilation.

mortem emphysematous lung, demonstrating collateral ventilation between segments and across the major fissure. Collateral ventilation after bronchial occlusion can be studied either by bronchoscopy [32-34] or by imaging techniques other than standard CT [35-37]. To the best of our knowledge, no reports of studies in which standard CT is used to assess collateral ventilation are available.
Another representative example of how $\Delta S V g$ maps can be used to assess collateral ventilation is shown in figure 7 . In this case, showing a patient different from that shown in figure 4, the application of a density mask ( $-970 \mathrm{HU}$ threshold) to the corresponding images taken at TLC and RV indicates a lower amount of tissue at RV than TLC in the dorsal region. On the $\Delta S V g$ map, this corresponds to negative (blue) $\Delta S V g$ values in 


\begin{tabular}{|c|c|c|c|c|c|c|c|c|c|c|c|c|}
\hline TABLE 1 & AA & C & TD & AA & C & TD & AA & C & TD & AA & C & TD \\
\hline Skewness & $-0.2 \pm 0.4$ & $-0.0 \pm 0.3$ & $-0.1 \pm 0.2$ & $0.0 \pm 0.7$ & $-0.1 \pm 1.1$ & $-0.1 \pm 0.5$ & $-0.2 \pm 0.4$ & $-0.1 \pm 0.4$ & $-0.003 \pm 0.3$ & $0.5 \pm 0.5^{* * *}$ & $0.6 \pm 0.5^{\star \star}$ & $0.6 \pm 0.3^{\star * *}$ \\
\hline QCV & $0.2 \pm 0.03$ & $0.3 \pm 0.04$ & $0.3 \pm 0.0$ & $1.5 \pm 0.6^{\star \star \star}$ & $1.7 \pm 1.0^{\star \star \star \star}$ & $1.2 \pm 0.5^{\star \star \star}$ & $0.2 \pm 0.04$ & $0.3 \pm 0.04$ & $0.3 \pm 0.03$ & $1.5 \pm 0.6^{\star \star \star}$ & $1.6 \pm 0.9 * \star \star$ & $1.2 \pm 0.5^{\star \star \star}$ \\
\hline
\end{tabular}

the same area, just below the lobar fissure. In this example, collateral ventilation seems to occur between lobes rather than within the same lobe. The general validity of the two representative cases reported above can be appreciated in figure 5 , where it is shown that all the patients show that a significant area of the histogram lies in the negative range. A possible way of quantifying the amount of collateral ventilation would therefore be to calculate the percentage of the $\Delta \mathrm{SVg}$ histogram lying below zero in a given region.

In the present work, we have also introduced a method for registration applied to CT lung images taken at different lung volumes, which extends previously proposed techniques. TORIGIAN et al. [38] introduced a registration algorithm to analyse density differences between different lung volumes in emphysema. The same authors proposed an image preprocessing algorithm, based on the image Laplacian, to overcome the requirement of grey intensity constancy of the OFM. In this study, we considered not only the image Laplacian for image pre-processing, but other features, such as the external border of the lung, the vessels and the fissures, which are anatomical structures that allow more accurate registration for spatially inhomogeneous functional differences within the same slice. Our development of these algorithms was necessary to allow registration of the images taken in the healthy subjects, where density changes between high and low lung volume are so high that the Laplacian is not sufficient to satisfy the requirements of OFM.

The main limitation of this work is that the analysis was performed in two dimensions. This is not a difficult problem when studying emphysema, as the small lung deformation can be tracked accurately in two dimensions. In the healthy lung, conversely, lung deformation is much larger and this can lead to areas without corresponding vessels in the images taken at TLC and RV. This error has been partially overcome by masking the vessels thresholding the images at $-400 \mathrm{HU}$ both at RV and TLC. Another limitation, due to the data available from the database of the clinical trial and healthy subjects, was to consider images reconstructed with different slice thicknesses between the volunteers $(5 \mathrm{~mm})$ and the patients $(10 \mathrm{~mm})$. Nevertheless, we have recently demonstrated [21] that the disproportionate effect of low-attenuation pixels on SVg can be significantly reduced by using thick slices $(5-10 \mathrm{~mm})$ combined with a smooth reconstruction filter. Another possible issue in our detailed quantitative CT analysis of severe emphysema is that accuracy of $\mathrm{SVg}$ is dependent on air calibration [19]. Nevertheless, the methods proposed in this study are based on differences between SVg values and, therefore, this possible limitation is overcome. Another slight limitation is the relatively modest number of patients; all patients were characterised by severe emphysema, which made a homogeneous group. The results obtained demonstrate high homogeneity within the groups and high statistical significance.

The application of the present work may have important clinical and physiological implications in the assessment of different stages of disease, and in the evaluation of either pharmacological or surgical treatments, such as minimally invasive interventions like transbronchial stents and endobronchial lung volume reduction. We believe that our method, once translated into clinical practice by a highly automated dedicated software, may be helpful both to identify regions (lobes and/or segments) where gas trapping is more pronounced and to distinguish those patients with and without collateral ventilation who are, therefore, more or less likely to benefit from lung volume reduction by minimally invasive interventions.

\section{SUPPORT STATEMENT}

This work was supported in part by NIH grant R01-HL090806 and Fondazione U. Veronesi.

\section{CLINICAL TRIAL}

This study is registered at www.clinicaltrials.gov with identifier number NCT00391612.

\section{STATEMENT OF INTEREST}

None declared. 
a)

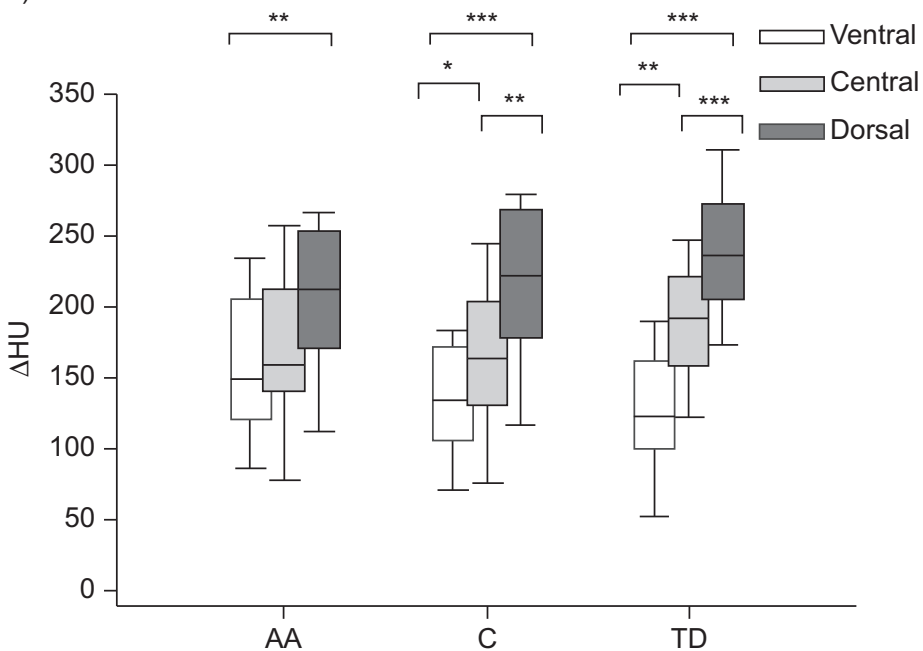

c)

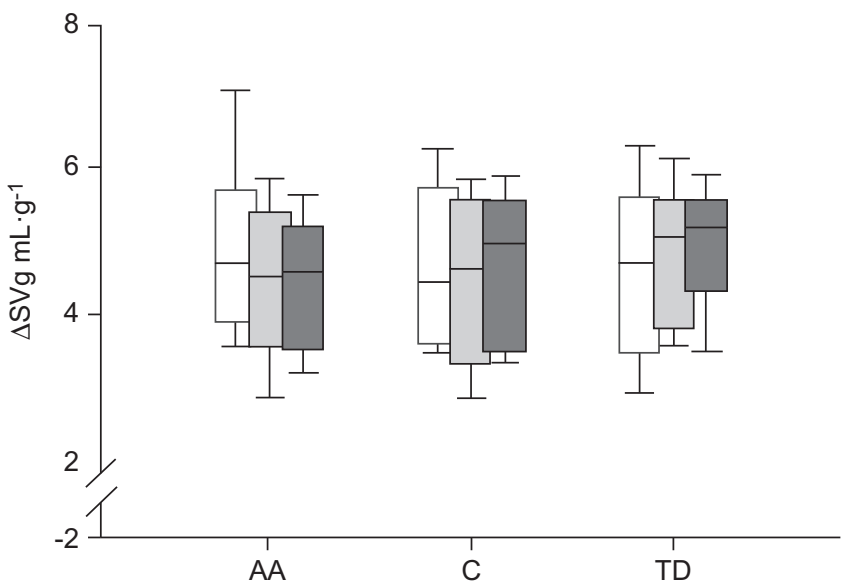

b)

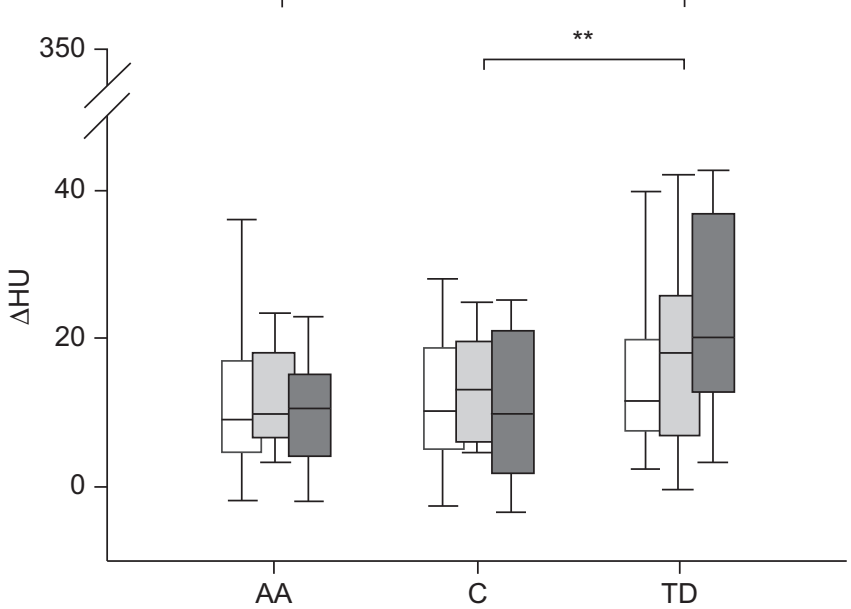

d)

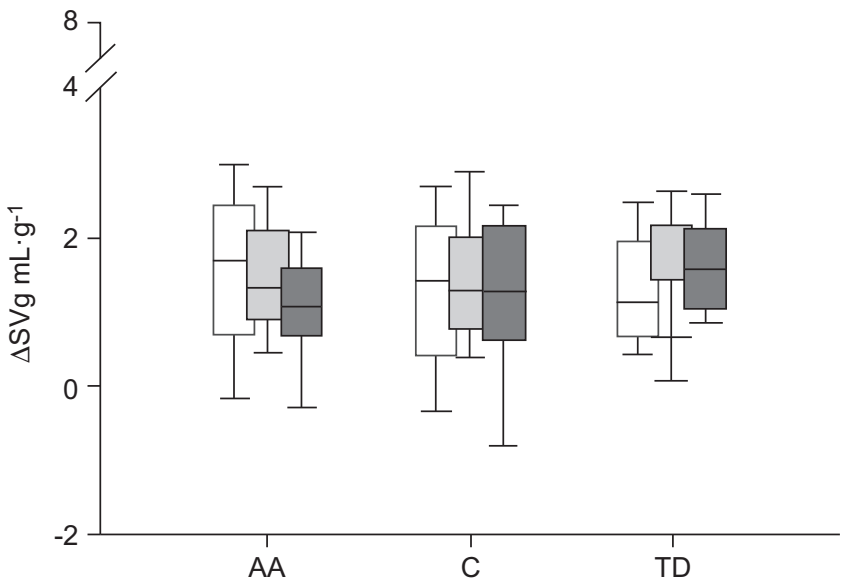

FIGURE 6. Gravity dependence of a and b) change in Hounsfield units ( $\Delta \mathrm{HU})$ and $\mathrm{c}$ and d) specific gas volume differnce ( $\Delta \mathrm{SVg}$ ) in a and c) healthy controls and b and d) emphysematous subjects. In each panel, the median values of ventral, central and dorsal portions of the lung at aortic arch (AA), carina (C) and top diaphragm (TD) levels are shown. ${ }^{*}: p<0.05 ; * *: p<0.01 ; * * *: p<0.001$.

a)

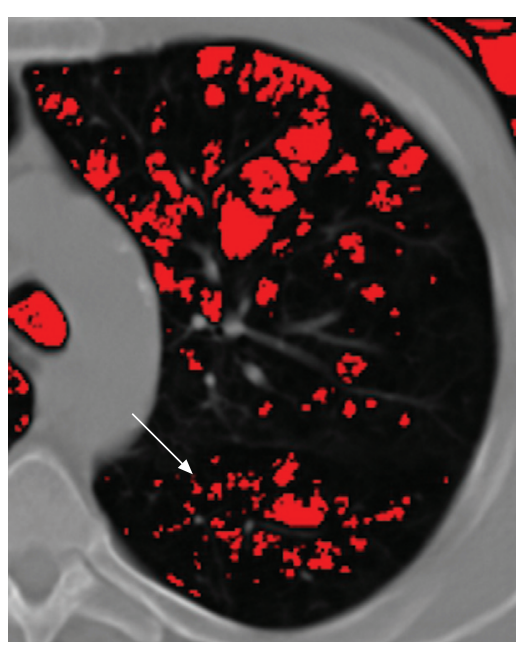

b)

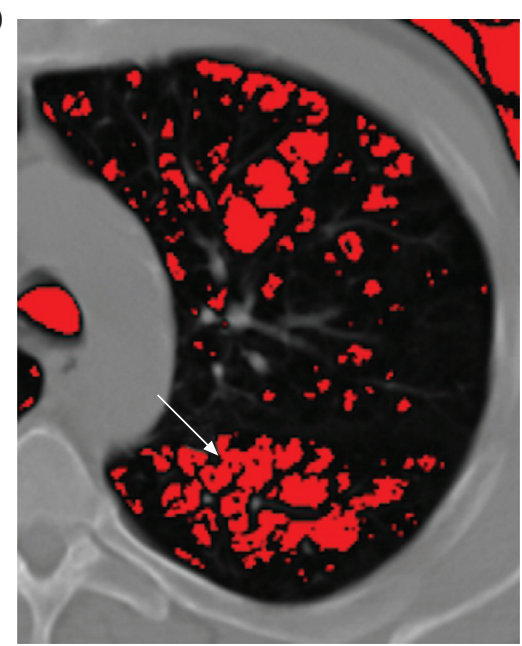

c)

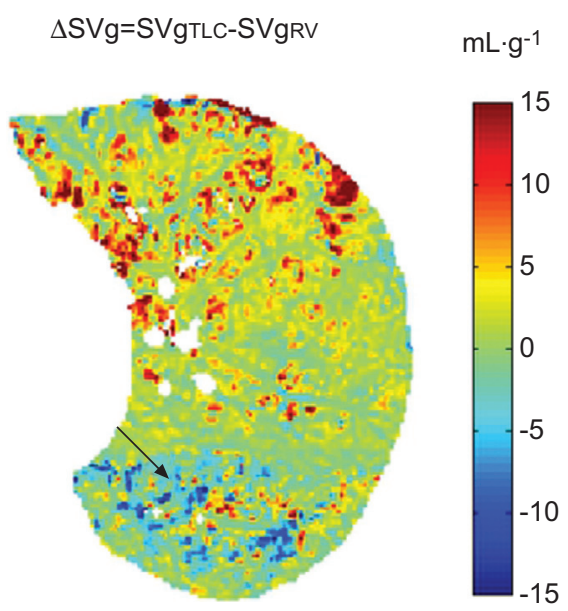

FIGURE 7. Example of interlobar collateral ventilation assessed by standard computed tomography. We applied a density mask to correspondent images at total lung capacity (TLC) and residual volume (RV) highlighting the pixels below -970 HU: a decrease in density from TLC to RV is clearly visible. The areas where this phenomena happens (e.g. arrows) result in negative change in specific gas volume $(\Delta \mathrm{SVg})$ values, shown in blue. 


\section{REFERENCES}

1 Choong CK, Macklem PT, Pierce JA, et al. Airway bypass improves the mechanical properties of explanted emphysematous lungs. Am J Respir Crit Care Med 2008; 178: 902-905.

2 Shah PL, Slebos DJ, Cardoso PF, et al. Bronchoscopic lung-volume reduction with Exhale Airway Stents for Emphysema (EASE trial): randomised, sham-controlled, multicentre trial. Lancet 2011; 378: 997-1005.

3 Wan IY, Toma TP, Geddes DM, et al. Bronchoscopic lung volume reduction for end-stage emphysema: report on the first 98 patients. Chest 2006; 129: 518-526.

4 Snell GI, Hopkins P, Westall G, et al. A feasibility and safety study of bronchoscopic thermal vapor ablation: a novel emphysema therapy. Ann Thorac Surg 2009; 88: 1993-1998.

5 Reilly J, Washko G, Pinto-Plata V, et al. Biological lung volume reduction: a new bronchoscopic therapy for advanced emphysema. Chest 2007; 131: 1108-1113.

6 Refaely Y, Dransfield M, Kramer MR, et al. Biologic lung volume reduction therapy for advanced homogeneous emphysema. Eur Respir J 2010; 36: 20-27.

7 Herth FJ, Eberhard R, Gompelmann D, et al. Bronchoscopic lung volume reduction with a dedicated coil: a clinical pilot study. Ther Adv Respir Dis 2010; 4: 225-231.

8 Milic-Emili J, Henderson JA, Dolovich MB, et al. Regional distribution of inspired gas in the lung. J Appl Physiol 1966; 21: 749-759.

9 Bunow B, Line B, Horton M, et al. Regional ventilatory clearance by xenon scintigraphy: a critical evaluation of two estimation procedures. J Nucl Med 1979; 20: 703-710.

10 Newman S, Pitcairn G, Hirst P, et al. Radionuclide imaging technologies and their use in evaluating asthma drug deposition in the lung. Adv Drug Deliv Rev 2003; 55: 851-867.

11 Lutey BA, Lefrak SS, Woods JC, et al. Hyperpolarized ${ }^{3} \mathrm{He} \mathrm{MR}$ imaging: physiologic monitoring observations and safety considerations in 100 consecutive subjects. Radiology 2008; 248: 655-661.

12 Fain SB, Korosec FR, Holmes JH, et al. Functional lung imaging using hyperpolarized gas MRI. J Magn Reson Imaging 2007; 25: 910-923.

13 Evans A, McCormack DG, Santyr G, et al. Mapping and quantifying hyperpolarized $3 \mathrm{He}$ magnetic resonance imaging apparent diffusion coefficient gradients. J Appl Physiol 2008; 105: 693-699.

14 Tajik JK, Tran BQ, Hoffman EA. Xenon enhanced CT imaging of local pulmonary ventilation. Proc SPIE 1996; 2790: 40-54.

15 Marcucci C, Nyahan D, Simon BA. Distribution of pulmonary ventilation using Xe-enhanced computed tomography in prone and supine dogs. J Appl Physiol 2001; 90: 421-430.

16 Simon BA. Non-invasive imaging of regional lung function using x-ray computed tomography. J Clin Monit Comput 2000; 16: 433-442.

17 Dougherty L, Torigian DA, Affusso JD, et al. Use of an optical flow method for the analysis of serial CT lung images. Acad Radiol 2006; 13: 14-23.

18 Dougherty L, Asmuth JC, Gefter WB. Alignment of CT lung volumes with an optical flow method. Acad Radiol 2003; 10: 249-254.
19 Coxson $\mathrm{HO}$, Rogers RM, Whittall KP, et al. A quantification of the lung surface area in emphysema using computed tomography. Am J Respir Crit Care Med 1999; 159: 851-856.

20 Salito C, Aliverti A, Gierada DS, et al. Quantification of trapped gas via CT and ${ }^{3} \mathrm{He}$ MRI in a new model of isolated airway obstruction. Radiology 2009; 253: 380-389.

21 Salito C, Woods JC, Aliverti A. Influence of CT reconstruction settings on extremely low attenuation values for specific gas volume calculation in severe emphysema. Acad Radiol 2011; 18: 1277-1284.

22 Mishima $\mathrm{M}$, Itoh $\mathrm{H}$, Sakai $\mathrm{H}$, et al. Optimized scanning conditions of high-resolution CT in the follow-up of pulmonary emphysema. J Comput Assist Tomogr 1999; 23: 380-384.

23 Lucas BD, Kanade T. An iterative image registration technique with an application to stereo vision. Proc IJCAI 1981; 81: 674-679.

24 Hedlund LW, Vock P, Effmann EL. Evaluating lung density by computed tomography. Semin Respir Med 1983; 5: 76-87.

$25 \mathrm{Hu}$ S, EA Hoffman, JM Reinhardt. Automatic lung segmentation for accurate quantification of Volumetric X-Ray CT Images. IEEE Trans Med Imaging 2001; 20: 490-498.

26 Horn BKP, Schunck G. Determining optical flow. Artif Intell 1981; 17: 185-203.

27 Beauchemin SS, Barron JL. The computation of optical flow. ACM Comput Surv 1995; 27: 433-467.

28 Bryan AC, Milic-Emili J, Pengelly D. Effect of gravity on the distribution of pulmonary ventilation. J Appl Physiol 1966; 21: 778-784.

29 Guerrero T, Sanders K, Noyola-Martinez J, et al. Quantification of regional ventilation from treatment planning CT. Int J Radiat Oncol Biol Phys 2005; 62: 630-634.

30 Guerrero $\mathrm{T}$, Sanders K, Castillo E, et al. Dynamic ventilation imaging from four dimensional computed tomography. Phys Med Biol 2006; 51: 777-791.

31 Hogg JC, Macklem PT, Thurlbeck WM. The resistance of collateral channels in excised human lungs. J Clin Invest 1969; 48: 421-431.

32 Terry PB, Traystman RJ, Newball HH. Collateral ventilation in man. N Engl J Med 1978; 298: 10-15.

33 Morrell NW, Wignall BK, Biggs T, et al. Collateral ventilation and gas exchange in emphysema. Am J Respir Crit Care Med 1994; 150: 635-641.

34 Aljuri N, Freitag L. Validation and pilot clinical study of a new bronchoscopic method to measure collateral ventilation before endobronchial lung volume reduction. J Appl Physiol 2009; 106: 774-783.

35 Salanitri J, Kalff V, Kelly M, et al. ${ }^{133}$ Xenon ventilation scintigraphy applied to bronchoscopic lung volume reduction techniques for emphysema: relevance of interlobar collaterals. Intern Med J 2005; 35: 97-103.

36 Bartel SE, Haywood SE, Woods JC, et al. Role of collateral paths in long-range diffusion in lungs. J Appl Physiol 2008; 104: 1495-1503.

37 Woods JC, Choong CK, Yablonskiy DA, et al. Hyperpolarized ${ }^{3} \mathrm{He}$ diffusion MRI and histology in pulmonary emphysema. Magn Reson Med 2006; 56: 1293-1300.

38 Torigian DA, Gefter WB, Affuso JD, et al. Application of an optical flow method to inspiratory and expiratory lung MDCT to assess regional air trapping: a feasibility study. AJR Am J Roentgenol 2007; 188: W276-W280. 